\title{
Discovering Novel Biomarkers for Pulmonary Hypertension
}

\author{
Nadiya P* \\ De Montfort University, Gateway House, Leicester, England \\ *Corresponding author: Nadiya Patel, De Montfort University, Gateway House, \\ Leicester, England, LE1 9BH.
}

\author{
Short Communication \\ Volume 1 Issue 1 \\ Received Date: July 19, 2018 \\ Published Date: October 11, 2018 \\ DOI: $10.23880 /$ aabsc- 16000105
}

\section{Short Communication}

Groundbreaking research into a new biomarker that can indicate cardiac damage and mortality in pulmonary hypertension patients has been identified in a recent study by Tzouvelekis, et al. on 'S100A12 [1] as a marker of worse cardiac output and mortality in pulmonary hypertension'. Biomarkers are objectively measured characteristics that provide an indication of normal biological processes, pathogenic processes or processes in the body that occur as a pharmaceutical response to treatment [2]. Findings of this study showed that biomarker S100A12 is elevated in pulmonary hypertension patients compared to healthy individuals [1]. This biomarker has emerged to be of value in various other inflammatory diseases, thus leading this study to be highly significant as results show that S100A12 is a determining factor and prognostic indicator of pulmonary hypertension.

Pulmonary hypertension is a form of cardiovascular disease in which there is a high blood pressure in the pulmonary arteries [3]. This disease was discovered in 1981 and due to the ongoing improvement of specialized therapeutic methods and diagnostic techniques, patient survival rate has increased from three [4] to ten years or more after initial diagnosis [5].

Advances in diagnostic techniques play a huge role in patient survival, as an earlier diagnosis can lead to faster treatment and reduced disease manifestation. The methodology used in this study involved the use of microarray analysis on peripheral blood mononuclear cells of pulmonary arterial hypertension patients, lungdisease associated pulmonary hypertension patients and healthy external control individuals. Results showed that both cohorts of patients had higher levels of S100A12 averaged at $32.6 \mathrm{ng} / \mathrm{mL}$, compared to the healthy individuals who had an average of $15.7 \mathrm{ng} / \mathrm{mL}$ [1]. The identification of S100A12 as a Molecular biomarker fits into the current research view as it refines prognostication and phenotyping of pulmonary hypertension patients.

There is sufficient evidence in this study to validate the findings of S100A12 as a biomarker of pulmonary hypertension. Other studies mention the discovery of novel biomarkers potentiating further improvements in diagnosis and treatment of patients with cardiovascular disease as a whole. This is because specific biomarkers and biomarker combinations can be directly correlated with pathogenic processes that may relate to a single or multiple types of cardiovascular disease [6]. A disadvantage is that if a biomarker is linked to multiple pathogenic processes, further testing will be required to pinpoint the exact disease present, and this may prolong disease diagnosis [7]. Regarding S100A12, it has also been linked to chronic heart failure, atherosclerosis [8] and familial Mediterranean fever [9].

The research may be criticized due to the recent timeframe of its publication. As it was published last month it may require further critical analysis by external academics. The results rely solely on the understanding of S100A12 working alone as a prognostic biomarker, however it may work alongside another to provide the data achieved. No further testing in this area was undertaken. As the results provided comparative concentration levels of the biomarker, validity was justified. The choice of blood specimen was easily accessible and presented minimal subject burden which could be logistically feasible if the study was repeated on a larger scale. Although molecular biomarkers provide a proximal measure of disease manifestation, other factors may be overlooked such as physiological factors, dietary factors and genetic variability. Physiological factors may contribute to the initial cause of disease and genetic variability could skew biomarker measures and give 
incorrect values, making an individual more susceptible to a disease.
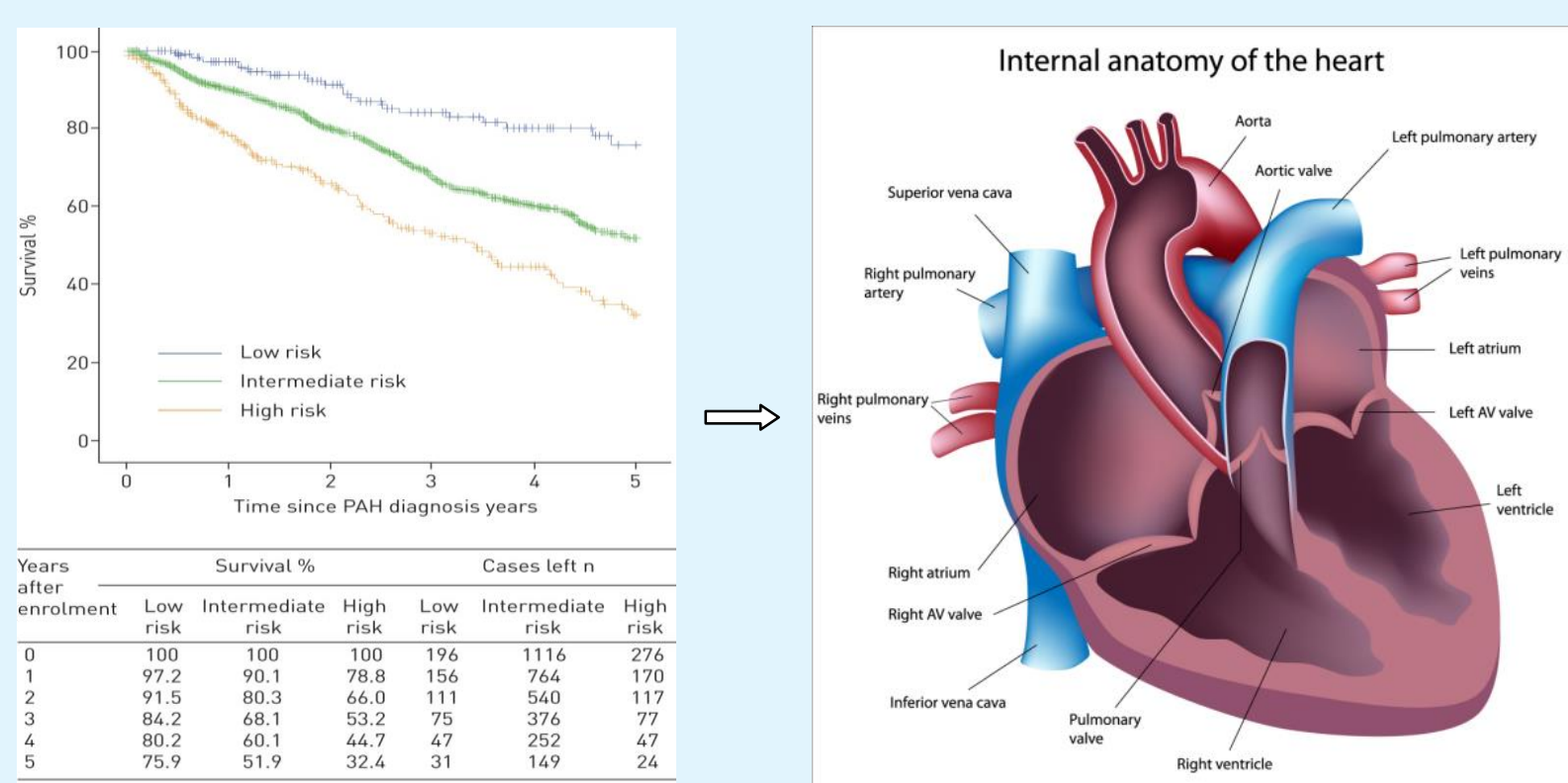

Figure 1: The comparison of three varying risk groups of patients with pulmonary hypertension. Interpretation leads to an understanding that in all three groups, an increase in disease diagnosis relates to a prolonged survival rate of pulmonary hypertension patients. Graphical comparison links to the image as a visual understanding of the manner in which pulmonary hypertension occurs. Heart anatomy has been edited to show this. Original data source: Marius, et al. [10].

\section{References}

1. Tzouvelekis A, Herazo-Maya J, Ryu C, Chu J, Zhang Y, et al. (2018) S100A12 as a marker of worse cardiac output and mortality in pulmonary hypertension. Respirology 23(8): 771-779.

2. Hong H, Goodsaid F, Shi L, Tong W (2010) Molecular biomarkers: a US FDA effort. Biomarkers in Medicine 4(2): 215-225.

3. Savale L, Guignabert C, Weatherald J, Humbert M (2018) Precision medicine and personalizing therapy in pulmonary hypertension: seeing the light from the dawn of a new era. European Respiratory Review 27(148): 180004.

4. Tonelli A, Arelli V, Minai O, Newman J, Bair N, et al. (2013) Causes and Circumstances of Death in Pulmonary Arterial Hypertension. American Journal of Respiratory and Critical Care Medicine 188(3): 365-369.
5. Wei-Ting C, Shih-Feng W, Chih-Hsin H, Jhih-Huan S, Jhi-Joung W (2016) Prognostic Factors in Patients With Pulmonary Hypertension-A Nationwide Cohort Study. Journal of the American Heart Association 5(9): e003579.

6. Bachmann K, Wang TJ (2017) Biomarkers of cardiovascular disease: contributions to risk prediction in individuals with diabetes. Diabetologia 61(5): 987-995.

7. Wilkinson J, Williams P, Yu W, Colan S, Mendez A, et al. (2018) Cardiac and inflammatory biomarkers in perinatally HIV-infected and HIV-exposed uninfected children. AIDS 32(10): 1267-1277.

8. Oesterle A, Bowman M (2015) S100A12 and the S100/Calgranulins Significance. Arteriosclerosis, Thrombosis, and Vascular Biology, 35(12): 24962507.

9. Kallinich T, Wittkowski H, Keitzer R, Roth J, Foell D (2009) Neutrophil-derived S100A12 as novel biomarker of inflammation in familial Mediterranean 


\section{Annals of Advanced Biomedical Sciences}

fever. Annals of the Rheumatic Diseases 69(4): 677682.

10. Marius H, Tilman K, Zixuan P, Benjamin N, Meyer K, et al. (2017) Mortality in pulmonary arterial hypertension: prediction by the 2015 European pulmonary hypertension guidelines risk stratification model. European Respiratory Journal 50(2): 1700740 .

(c) (1) 